\title{
Optimal Estimation
}

\author{
Jorma Rissanen
}

Helsinki Institute for Information Technology and Tampere University of Technology, Finland

\section{Modeling Problem}

Data $Y=\left\{y_{t}: t=1,2, \ldots, n\right\}$, or $Y \mid X=\left\{\left(y_{t}, x_{1, t}, x_{2, t}, \ldots\right)\right\}, X$ explanatory variables. Want to learn properties in $Y$ expressed by set of distributions as models: $f\left(Y \mid X_{s} ; \theta, s\right)$, where $\theta=\theta_{1}, \ldots, \theta_{k(s)}$ real-valued parameters, $s$ structure parameter: for picking the most important variables in $X$.

\subsection{Models and Estimators}

To simplify notations write $y_{t}, x_{1, t}, x_{2, t}, \ldots$ as $x_{t}$; structures determined by number $k$ of real-valued parameters.

Classes of parametric models

$$
\begin{aligned}
\mathcal{M}_{k} & =\left\{f\left(x^{n} ; \theta, k\right): \theta \in \Omega^{k} \subset R^{k}\right\} ; k \leq n \\
\mathcal{M} & =\left\{\mathcal{M}_{k}: k=1,2, \ldots, K, K \leq n\right\} .
\end{aligned}
$$

Sets of estimator functions $\bar{\theta}(\cdot), \bar{k}(\cdot)$. Consider the distributions defined by estimators

$$
\begin{aligned}
& \text { for fixed } k: \bar{f}\left(x^{n} ; k\right)=f\left(x^{n} ; \bar{\theta}\left(x^{n}\right), k\right) / \bar{C}_{k, n} \\
& \bar{C}_{k, n}=\int f\left(y^{n} ; \bar{\theta}\left(y^{n}\right), k\right) d y^{n} \\
& \text { in general : } \bar{f}\left(x^{n}\right)=\bar{f}\left(x^{n} ; \bar{k}\left(x^{n}\right)\right) / \bar{C}_{n} \\
& \bar{C}_{n}=\sum_{k} \int_{\bar{k}\left(y^{n}\right)=k} \bar{f}\left(y^{n} ; k\right) d y^{n}
\end{aligned}
$$

Let $\hat{\theta}(\cdot), \hat{k}(\cdot)$ be the estimator that maximizes $\bar{C}_{n}$ :

$$
\hat{C}_{n}=\max _{\bar{\theta}(\cdot), \bar{k}(\cdot)} \bar{C}_{n}
$$

It also maximizes the probability or density $\hat{f}\left(x^{n}\right)$ on the observed data, which is taken as the single postulate for this theory of estimation. The maximum $\hat{C}_{n}$ is called the maximum capacity, and it is also the maximum mutual information that any estimator can obtain about the models in the class. 